\title{
Geographic Routing Oriented Sleep Scheduling in Duty-Cycled Mobile Sensor Networks
}

\author{
Sukanya $\mathbf{N}^{1}$, Sudha $\mathbf{R}^{2}$ \\ ME-Communication Systems, Sri Shakthi Institute of Engineering and Technology, Coimbatore, India ${ }^{1}$ \\ Assistant Professor, Department of ECE,Sri Shakthi Institute of Engineering and Technology, Coimbatore, India ${ }^{2}$
}

\begin{abstract}
In this paper, we focus on sleep scheduling for geographic routing in duty-cycled WSNs with mobile sensors and propose two geographic-distance-based connected-k neighborhood (GCKN) sleep scheduling algorithms. The first one is the geographic-distance-based connected-k neighborhood for first path (GCKNF) sleep scheduling algorithm. The second one is the geographic-distance-based connected-k neighborhood for all paths (GCKNA) sleep scheduling algorithm. By theoretical analysis and simulations, we show that when there are mobile sensors, geographic routing can achieve much shorter average lengths for the first transmission path explored in WSNs employing GCKNF sleep scheduling and all transmission paths searched in WSNs employing GCKNA sleep scheduling compared with those in WSNs employing mobile sensors using Weighted Rendezvous Planning (WRP).
\end{abstract}

Keywords: Connected- $k$ neighborhood (CKN), duty-cycle, geographic routing, mobility, wireless sensor networks (WSNs).

\section{INTRODUCTION}

Geographic routing is one of the most promising routing schemes in wireless sensor networks (WSNs) due to its simplicity, scalability, and efficiency.

In such a scheme, regardless of the network size, the forwarding decision is determined purely based on the location of each node and it can be done even when there are irregular radio ranges and localization errors.

However, nearly all these works overlook one important fact that sensors can actually be mobile to gain better energy efficiency, channel capacity, etc., and enable a lot of new application scenarios.

For example, because sensors can move, they can transmit their data from different locations and avoid the problem that sensors near the gateway or sink always exhaust their energy first; thus, energy usage can be more efficient.

Also, mobile sensors such as mobile phones or cars can become the interface between the information center and the mobile customers; thus, real-time information (e.g., traffic information) transmitted from the information center to these mobile objects can be provided to nearby customers.

Recently, the research focus of geographic routing is centering on WSNs with duty-cycles, since dutycycled WSNs have a natural advantage of saving energy by dynamically putting nodes to sleep and waking them according to some sleep scheduling algorithms.

This paper addresses the sleep scheduling problem in dutycycled WSNs with mobile nodes employing geographic routing. We propose two geographic-distancebased connected-k neighborhood (GCKN) sleep scheduling algorithms. The first one is the geographic- distance-based connected-kneighborhood for first path1 (GCKNF) sleep scheduling algorithm, aiming at geographic routing utilizing only the first transmission path in duty-cycled mobile WSNs.

The second one is the geographic-distance-based connected $\mathrm{k}$ neighborhood for all paths2 (GCKNA) sleep scheduling algorithm, for geographic routing concerning all paths explored in duty-cycled mobile WSNs.

The main contributions of this paper are summarized as follows.

1)This paper is proposing and analyzing sleep scheduling algorithms for geographic routing in duty-cycled mobile WSNs, which take full advantages of both duty cycling and sensor mobility.

2)This paper proposes two GCKN algorithms, GCKNF and GCKNA sleep scheduling algorithms. The GCKNF sleep scheduling algorithm is designed to explore shorter first transmission paths for geographic routing in dutycycled mobile WSNs.

3)The GCKNA sleep scheduling algorithm aims at shortening all routing paths for multipath transmissions in duty-cycled mobile WSNs. These GCKN algorithms incorporate the connected- $k$ neighborhood requirement and geographic routing requirement to change the asleep or awake state of sensor nodes.

\section{Geographic Routing}

The basic idea of geographic routing is greedy routing. Specifically, each packet is tagged with the coordinates of its destination, all nodes know their own coordinates, and a node forward the packet to its neighbor that is geographically closest to the destination. 


\section{Sleep Scheduling}

The basic mechanism for sleep scheduling is to select a subset of nodes to be awake in a given epoch while the remaining nodes are in the sleep state that minimizes power consumption, so that the overall energy consumption can be reduced.

\section{EXISTING SYSTEM}

In the existing method, a hybrid unconstrained movement pattern for a mobile sink with the aim of balancing the energy consumption of sensor nodes called WRP.

We prefer nodes that have a high degree. This is critical as sensor nodes in dense parts of a WSN generate the highest number of packets.

\section{WEIGHTED RENDEZVOUS PLANNING}

WRP preferentially designates sensor nodes with the highest weight as a RP.

The weight of a sensor node is calculated by multiplying the number of packets that it forwards by its hop distance to the closest RP on the tour. Thus, the weight of sensor node $i$ is calculated as

$W i=\operatorname{NFD}(i) \times H(i, M)(1)$

Based on (1), sensor nodes that are one hop away from an RP and have one data packet buffered get the minimum weight.

Hence, sensor nodes that are farther away from the selected RPs or have more than one packet in their buffer have a higher priority of being recruited as an RP.

Hence, visiting the highest weighted node will reduce the number of multi-hop transmissions and thereby minimizes the energy consumption.

Fig. 1 shows an example of how WRP finds a traveling tour for a mobile sink. The maximum tour length is $l \max =$ $90 \mathrm{~m}$. WRP starts from the sink node and adds it to the tour, i.e., $M=[$ Sink $]$.

Then, an SPT rooted at the sink node is constructed.[Fig. 1(a)]. In the first iteration, WRP adds node 10 to the tour because it has the highest weight, yielding $M=$ [Sink, 10].

As Fig. 1(b) shows, the tour length of $M$ is smaller than the required tour length $(56<90)$, meaning node 10 stays in the final tour (lines 22-32).

In the second iteration, WRP recalculates the weight of sensor nodes because node 10 is now part of the tour.

In this iteration, WRP selects node 6 as the next RP, which has the highest weight.

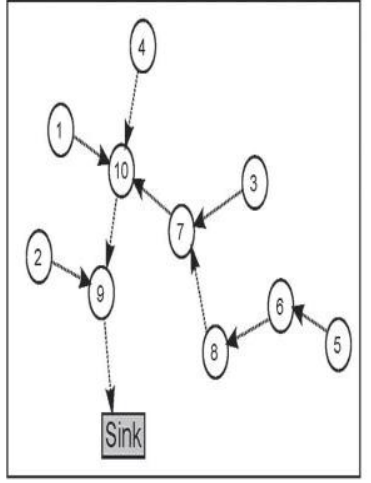

(a)

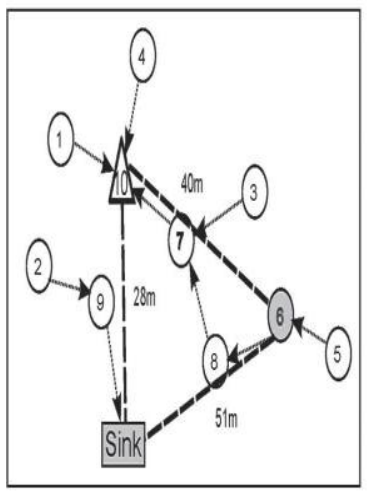

(c)

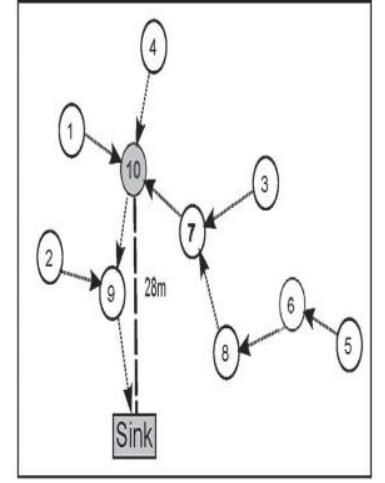

(b)

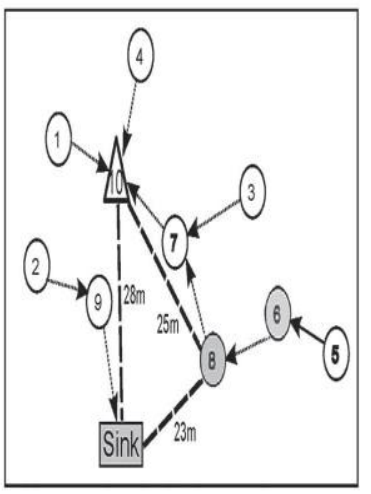

(d)

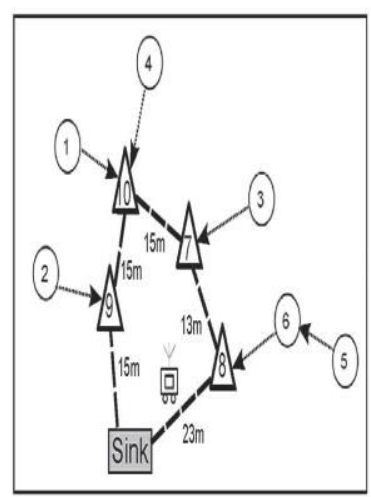

(e)

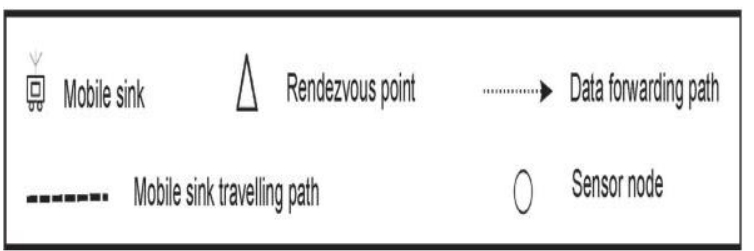

Fig 1 Example of WRP operating in a WSN with ten nodes.

As Fig. 1(c) shows, the tour length of $M=[$ Sink, 10,6] is larger than the required tour length $(119>90)$. Consequently, WRP removes node 6 from the tour $M=$ [Sink, 10].In the third iteration, the weight of sensor nodes will not change because node 6 is not selected as an RP but it stays marked and will not be selected.WRP selects node 8 because it has the highest weight and is not marked . The TSP function returns $76 \mathrm{~m}$ for $M=[$ Sink, 10, 8], 
which is less than $90 \mathrm{~m}$. Therefore, node 8 is added to the tour. The process continues, yielding a final tour of $M=$ [Sink, 8, 7, 10, 9] with a tour length of $81 \mathrm{~m}$, which is less than the required tour length [see Fig. 1(e)].

\section{PROPOSED SYSTEM}

We consider the following six factors for both GCKNF and GCKNA.

1. A node should go to sleep assuming that at least $k$ of its neighbors will remain awake so as to save energy as well as keep it $k$-connected.

2. The asleep or awake state of nodes should be allowed to change between epochs so that all nodes can have the opportunity to sleep and avoid staying awake all the time, thus distributing the sensing, processing, and routing tasks across the network to prolong the network lifetime.

3. Although each node decides to sleep or wake up locally, the whole network should be globally connected so that data transmissions can be performed.

4. Each node should have enough initial neighbors in order to make it easier for the node to satisfy the connected- $k$ neighborhood requirement; thus, it is more likely to be asleep after sleep scheduling. For GCKNF, which emphasizes the first transmission path of geographic routing, we further take the following factor into account.

5. The neighbor of each node, which is closest to sink, should be awake so that geographic routing can utilize these nearest neighbor nodes to make the first transmission path as short as possible. For GCKNA, which considers all transmission paths, we further take the following factor into consideration.

6. For each node, as many as possible of its neighbor nodes that are closer to the sink should be awake so that geographic routing can make all transmission paths as short as possible.

\section{Pseudocode of GCKNF algorithm}

First: Run the following at each node $u$.

1) Send probe packet $p_{u}$ to neighbors and receive the ack packet.

2) Compute whether $u$ 's current neighbors $C N_{u} \geq$ $\min \left(k, d_{u}\right)$.

3) Maintain its transmission radius if the above the condition holds or its current transmission radius is maximum.

Otherwise, increase its transmission radius until $C N_{u} \geq \min \left(k, d_{u}\right)$.

Second: Run the following at each node $u$.

1) Get its geographic location $g_{u}$ and sink location $g_{s}$.

2) Broadcast $g_{u}$ and receive the geographic locations of its all neighbors $A_{u}$. Let $G_{u}$ be the set of these geographic locations.

3) Unicast a flag to $w, w \in A_{u}$ and $g_{w}$ is the closest to sink in $G_{u}$.

Third: Run the following at each node $u$.

1) Pick a random rank rank

2) Broadcast rank $k_{u}$ and receive the ranks of its currently awake neighbors $N_{u}$. Let $R_{u}$ be the set of these ranks.
3) Broadcast $R_{u}$ and receive $R_{v}$ from each $v \in N_{u}$.

4) If $\left|N_{u}\right|<k$ or $\left|N_{v}\right|<k$ for any $v \in N u$, remain awake.

Return.

5) Compute $C u=\{v \mid v \in N u$ and rankv < ranku $\}$.

6) Go to sleep if the following three conditions hold. Remain awake otherwise

- Any two nodes in $C u$ are connected either directly themselves or indirectly through nodes within u's two-hop neighborhood that have rank less than ranku.

- Any node in $\mathrm{Nu}$ has at least $k$ neighbors from $\mathrm{Cu}$.

- It does not receive a flag.

7) Return.

\section{Pseudocode of GCKNA algorithm}

First: Run the following at each node $u$.

1) Send probe packet $p u$ to neighbors and receive the ack packet.

2) Compute whether $u$ 's current neighbors $\mathrm{CNu}>$ $\min (k, d u)$

3) Maintain its transmission radius if the above condition holds or its current transmission radius is the maximum. Otherwise, increase its transmission radius until $C N u \geq \min (k, d u)$.

Second: Run the following at each node $u$.

1) Get its geographic location $g u$ and sink location gs. Further get the geographic distance between itself and sink granku.

2) Broadcast granku and receive the geographic distance ranks of its currently awake neighbors $N u$.Let $R u$ be the set of these ranks.

3) Broadcast $R u$ and receive $R v$ from each $v \in N u$.

4) If $|N u|<k$ or $|N v|<k$ for any $v \in N u$, remain awake.

Return.

5) Compute $C u=\{v \mid v \in N u$ and grankv < granku $\}$.

6) Go to sleep if both the following conditions hold. Remain awake otherwise.

- Any two nodes in $\mathrm{Cu}$ are connected either directly themselves or indirectly through nodes within u's two-hop neighborhood that have grank less than granku.

- Any node in $N u$ has at least $k$ neighbors from $\mathrm{Cu}$.

7) Return.

\section{Analysis of GCKN Algorithms}

Theorem:

Node $u$ will have at least $\min (k, o u)$ awake neighbors after running GCKN algorithms, if it has ou neighbors in the original network.

Proof:

If $o u<k$, all of $u$ 's neighbors should keep awake (Step 4 of the third part of GCKNF or Step 4 of the second part of GCKNA) and the node will have ou awake neighbors. Otherwise, when $o u \geq k$, we prove the theorem by contradiction. Suppose that node $u$ will not have at least $k$ awake neighbors after running GCKN algorithms, i.e., we can assume that the $i$ 'th lowest ranked (for GCKNF) or 
granked (for GCKNA) neighbor $v$ of $u, i \leq k$, decides to sleep. Then $C u$ will have at most $i-1$ nodes that are neighbors of $u$. But since $i-1<k, v$ cannot go to sleep according to the Step 6 of third part of GCKNF or Step 6 of second part of GCKNA. This is a

contradiction. In other words, the $k$ lowest granked neighbors of $u$ will all remain awake after running the algorithm, and hence, $u$ will have at least $k$ awake neighbors.

\section{Theorem 2 .}

Running GCKN algorithms produces a

connected-network if the original network is connected.

Proof:

We prove this theorem by contradiction.

Assuming that the output network after running GCKN algorithms is not connected. Then, we put the deleted nodes (asleep nodes decided by GCKN algorithms) back in the network in ascending order of their ranks (for GCKNF) or granks (for GCKNA), and let $u$ be the first node that makes the network connected again. Note that by the time we put $u$ back, all the members of $C u$ are already present and nodes in $C u$ are already connected since they are connected by nodes with rank < ranku (for GCKNF) or grank < granku (for GCKNA). Let $v$ be a node that was disconnected from $C u$ but now gets connected to $C u$ by $u$. But this contradicts the fact that $u$ can sleep only if all its neighbors (including $v$ ) are connected to $\geq k$ nodes in $C u$ (Step 6 of third part of GCKNF or Step 6 of second part of GCKNA).

\section{Theorem:}

GCKN sleep scheduling-based WSN can provide as short as possible transmission path explored by geographic routing when there are mobile sensor nodes.

\section{Proof:}

We prove this by analyzing the resultant topology after running GCKNF or GCKNA. Concerning GCKNF, given that there is a network Ngcknf resulting from GCKNF, based on the algorithm presentation of GCKNF, we can deduce that the neighbor node that is closest to the sink for any node,will be among the awake nodes of the Ngcknf (Step 6 of the third part of GCKNF). In other words, no matter which node the geographic routing chooses to be the first forwarding node, all successor nodes closest to sink can be utilized by the geographic routing. Thus, the length of the first transmission path explored by geographic routing can be as short as possible.

Regarding GCKNA, assume that there is a network Ngckna created by GCKNA. From the algorithm description of GCKNA, we can determine that for any node, say $u$, if it determines to be asleep, it must make sure that either

1) its all awake 1-hop neighbor nodes are connected by themselves with grank < granku or connected by their two-hop neighbor nodes with grank < granku;

2) any of its awake one-hop neighbor nodes should have at least $k$ neighbor nodes from the subset of the one-hop neighbor nodes with grank < granku (Step 6 of the second part of GCKNA). This means that compared with the asleep nodes, the awake nodes generally have closer geographic distance to the sink

In other words, geographic routing can have access to as many as possible closer neighbor nodes to the sink under the priority of network connectivity after sleep scheduling. Thus, the length of all transmission paths searched by geographic routing can also be as short as possible.

\section{IVSIMULATION RESULTS}

We compare the results of sleep algorithms with the existing Weighted Rendezvous Planning (WRP) and Cluster Based (CB) simulated using NS2.

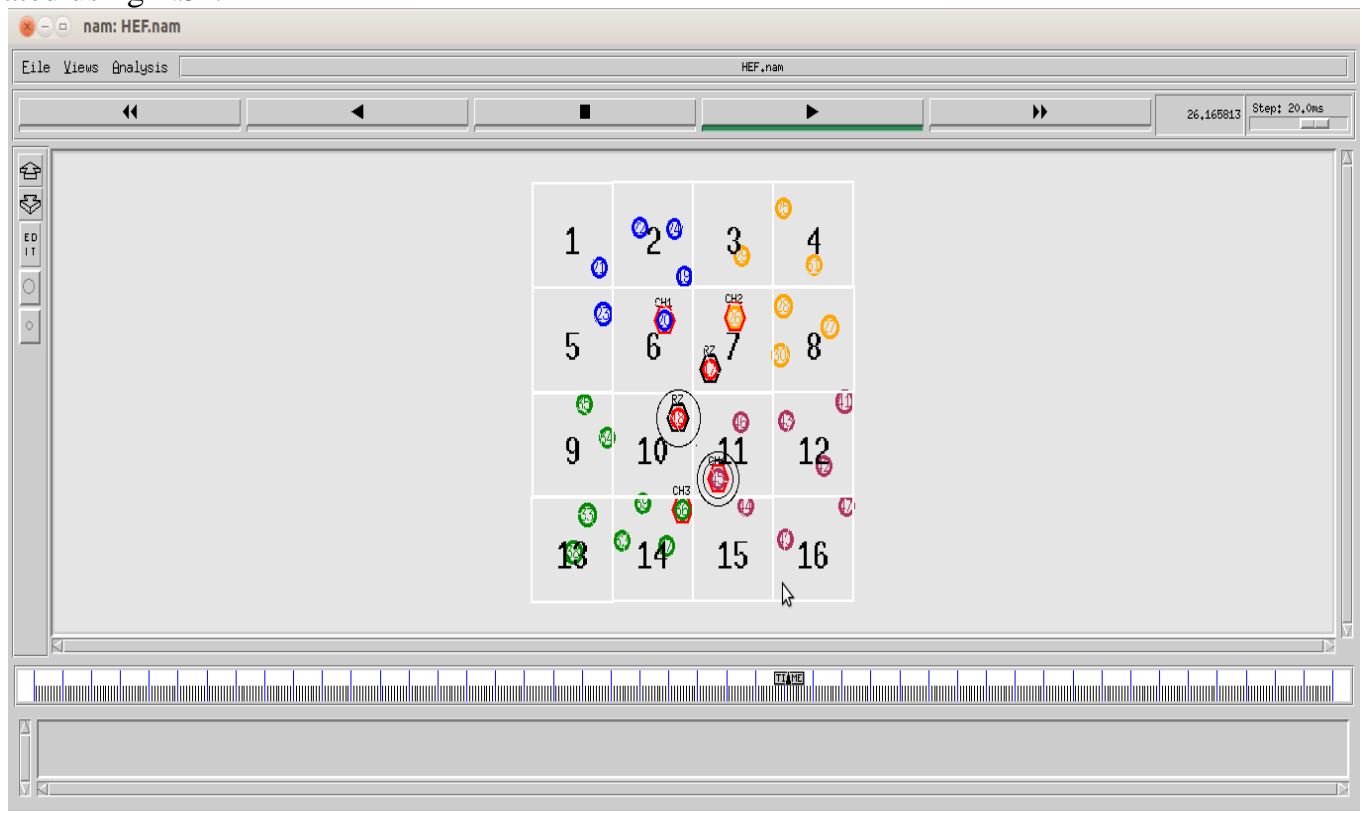

Fig 2 Simulation result of connected-k neighborhood for first path (GCKNF) sleep scheduling algorithm 


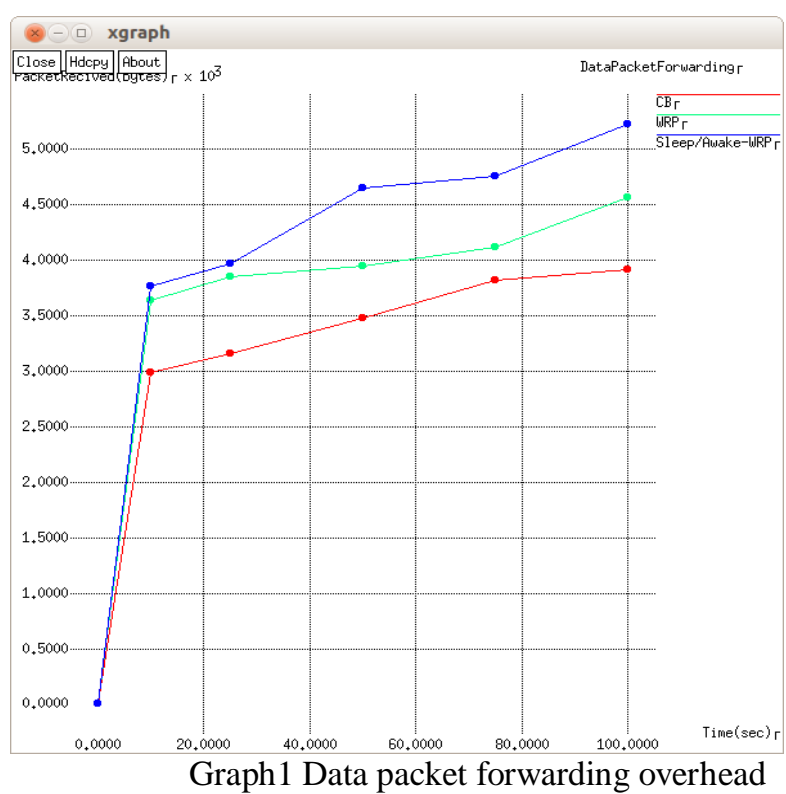

From the graph1 it is clear that the packet forwarding is greater for the proposed than the existing methods. From the graph2 it is clear that the delay is lesser for the proposed method when compared with the existing methods.

\section{CONCLUSION}

In this paper, we have explored geographic routing in duty-cycled mobile WSNs and proposed two geographicdistance based connected- $k$ neighborhood (GCKN) sleep scheduling algorithms for geographic routing schemes to be applied into duty-cycled mobile WSNs which can incorporate the advantage of sleep scheduling and mobility. The first geographic-distance based connected- $k$ neighborhood for first path (GCKNF) sleep scheduling algorithm minimizes the length of first transmission path explored by geographic routing in duty-cycled mobile WSNs. The second geographic-distance based connected$k$ neighborhood for all paths (GCKNA) sleep scheduling algorithm reduces the length of all paths searched by geographic routing in duty-cycled mobile WSNs. Our work has shown that sleep scheduling is a worthy research direction to adapt geographic forwarding methods into duty-cycled mobile WSNs.

\section{ACKNOWLEDGEMENT}

First of all we sincerely thank the almighty who is most beneficent and merciful for giving us knowledge and courage to complete the seminar work successfully. We also express our gratitude to all the teaching and nonteaching staff of the college especially to our department for their encouragement and help done during our work. Finally, we appreciate the patience and solid support of our parents and enthusiastic friends for their encouragement and moral support for this effort.

\section{REFERENCES}

[1] B. Leong, B. Liskov, and R. Morris, "Geographic routing without planarization," in Proc. NSDI, 2006, pp. 339-352.

[2] Y.-J. Kim, R. Govindan, B. Karp, and S. Shenker, "Lazy cross-link removal for geographic routing," in Proc. SenSys, 2006, pp. 112-124.

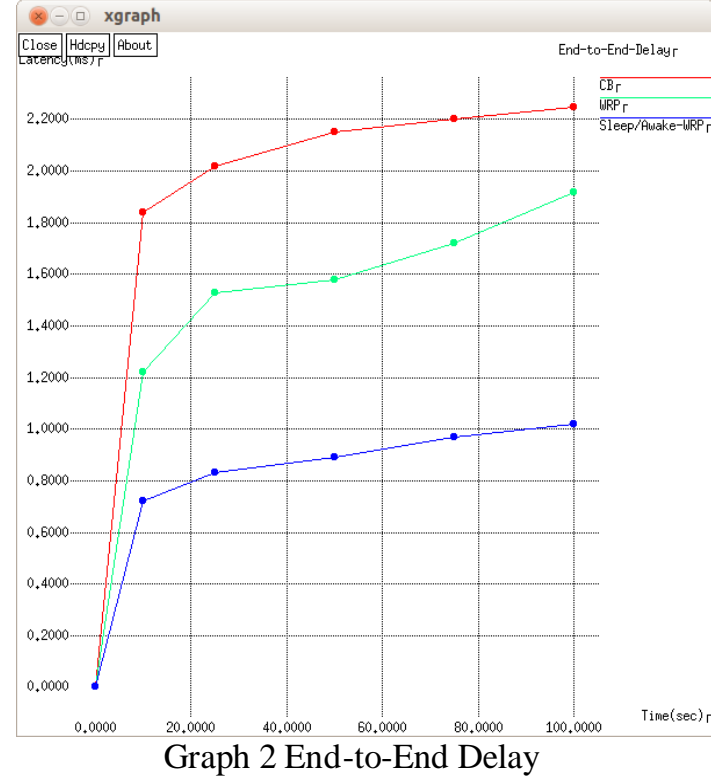

[3] L. Zhang and Y. Zhang, "Energy-efficient cross-layer protocol of channel aware geographic-informed forwarding in wireless sensor networks," IEEE Trans. Veh. Technol., vol. 58, no. 6, pp. 30413052, Jul. 2009.

[4] C.-F. Hsin and M. Liu, "Network coverage using low duty-cycled sensors: Random \& coordinated sleep algorithms," in Proc. IPSN, 2004, pp. 433-442.

[5] K. P. Naveen and A. Kumar, "Tunable locally-optimal geographical forwarding in wireless sensor networks with sleepwake cycling nodes," in Proc. IEEE INFOCOM, 2010, pp. 1-9.

[6] Q. Fang, J. Gao, and L. J. Guibas, "Locating and bypassing routing holes in sensor networks," in Proc. IEEE INFOCOM, 2004, pp. 2458-2468.

[7] L. Shu et al., "TPGF: Geographic routing in wireless multimedia sensor networks,” Telecommun. Syst., vol. 44, no. 1/2, pp. 79-95, Jun. 2010.

[8] C. Ma et al., "A geographic routing algorithm in duty-cycled sensor networks with mobile sinks," in Proc. MSN, 2011, pp. 343-344.

\section{BIOGRAPHY}

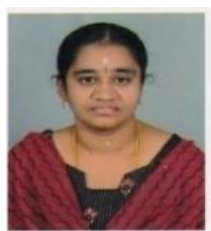

Sukanya did her Bachelor of Engineering in Electronics and Communication Engineering at Vivekanandha College of Engineering for Women, Tiruchengode and doing Master of Engineering in Communication Systems at Sri Shakthi Institute of Engineering and Technology, Coimbatore, India. Her research interests include Sensor Network, Communication System Design. She has presented two papers in International Conference, and presented a project in Kumaraguru college of technology, Coimbatore and published three International journals 